\title{
FORMATOS AUDIOVISUALES EMERGENTES PARA MOOCS: DISEÑO INFORMATIVO, EDUCATIVO Y PUBLICITARIO
}

\author{
Emerging audiovisual formats for MOOCs: \\ Informative, educative and advertising design
}

\section{Mario Rajas, Belén Puebla-Martínez y Miguel Baños}

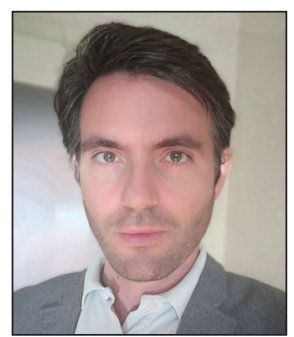

Mario Rajas, doctor en Comunicación Audiovisual por la Universidad Complutense de Madrid, es profesor en la Facultad de Comunicación de la Universidad Rey Juan Carlos (URJC). Editor de Icono14 Editorial y coordinador de la Unidad de Producción de Contenidos Académicos de la URJC. Su área de investigación es la narrativa, la estética y la tecnología de los medios audiovisuales. Ha publicado artículos científicos, monografías y manuales docentes y ha realizado estancias de investigación en universidades como el Tecnológico de Monterrey (México) o la University of California Los Angeles (EUA).

https://orcid.org/0000-0002-3807-8327

mario.rajas@urjc.es

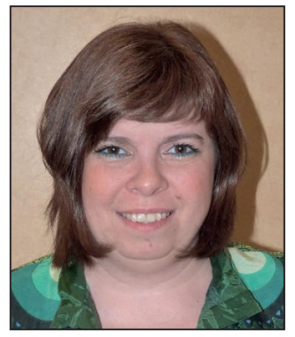

Belén Puebla-Martínez, doctora en Ciencias de la Comunicación por la Universidad Rey Juan Carlos (URJC), licenciada en Periodismo (2005) y en Comunicación Audiovisual (2007) por la misma universidad, es profesora visitante de la URJC. Es directora del grupo de investigación Visual. Investigando la comunicación en España. Sus líneas de investigación se centran en el diseño de la educación mediática y las competencias digitales, entre otras. Primer premio en la III Edición de profesores innovadores de la URJC (2016). Es autora de publicaciones sobre diseño entre las que destaca The mapmaker who saved a newpaper" en 22ㅇ Premios internacionales de infografía Malofiej.

https://orcid.org/0000-0002-1481-4238

belen.puebla@urjc.es

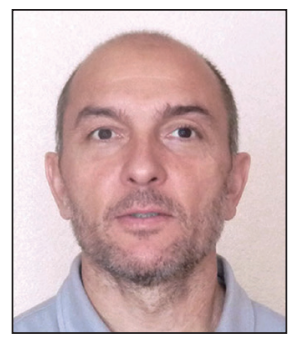

Miguel Baños es doctor en Ciencias de la Información por la Universidad Complutense de Madrid y profesor titular en la Universidad Rey Juan Carlos (URJC) en el área de Comunicación Audiovisual y Publicidad. Cuenta con una amplia experiencia docente e investigadora. Ha participado en proyectos de investigación financiados en convocatorias públicas, nacionales e internacionales. Autor de libros, capítulos de libro y artículos (siempre en temas de comunicaciones de marketing) tanto en revistas científicas como profesionales. Durante casi veinte años ha sido creativo publicitario en agencias nacionales e internacionales.

https://orcid.org/0000-0002-0195-2754

miguel.banos@urjc.es

Universidad Rey Juan Carlos Facultad de Ciencias de la Comunicación, Departamento de CC. de la Comunicación y Sociología Edificio Departamental I Camino del Molino, s/n. 28934 Fuenlabrada (Madrid), España

\section{Resumen}

Los formatos audiovisuales son esenciales en la enseñanza virtual. Los vídeos en cursos MOOC ejercen simultáneamente una función informativa, educativa y publicitaria en la programación del curso y en las redes sociales. En este trabajo se estudia el formato de los vídeos para cursos y se hace una valoración crítica seguida de propuestas de mejora. Se analizan los vídeos de 50 webs de cursos virtuales de la plataforma edX desde el punto de vista del diseño narrativo del contenido y de su realización. Los resultados obtenidos muestran que los vídeos para MOOC tienen estructura de lección magistral y códigos visuales y sonoros típicos de la clase presencial. Se formulan soluciones para incorporar a tales vídeos la potencialidad creativa de lenguajes y tecnologías audiovisuales usados en cine y televisión.

\section{Palabras clave}

Enseñanza virtual; MOOC; Formatos audiovisuales; Narrativa; Lenguajes audiovisuales; Publicidad. 


\begin{abstract}
Audiovisual formats are essential for virtual learning. Videos in MOOC courses exercise an informative, educational, and advertising function in the course program and in social networks. In this article, videos for courses are reviewed critically and include proposals for improvement. Videos of 50 courses of the $e d X$ platform are analyzed from the point-of-view of the narrative design of their content and audiovisual components. The results show that MOOC videos have a master lesson structure, with the visual and sound codes of classroom lectures. Solutions that incorporate creative languages and audiovisual technologies used in film and television are proposed.
\end{abstract}

\title{
Keywords
}

Virtual learning; Online learning; MOOCs; Audiovisual formats; Videos; Storytelling; Audiovisual languages; Advertising.

Rajas, Mario; Puebla-Martínez, Belén; Baños, Miguel (2018). “Formatos audiovisuales emergentes para MOOCs: diseño informativo, educativo y publicitario”. El profesional de la información, v. 27, n. 2, pp. 312-321.

https://doi.org/10.3145/epi.2018.mar.09

\section{Introducción}

La enseñanza universitaria semipresencial $u$ online ha incorporado las producciones audiovisuales como un recurso fundamental de su modelo de aprendizaje virtual (Woolfitt, 2015; Kaltura, 2016; iSpring, 2016). Formatos estandarizados provenientes de los medios de comunicación de masas como el vídeo o el podcast se han convertido, gracias a la incorporación y expansión de las tecnologías emergentes, en materiales recurrentes en la impartición de titulaciones de grado o posgrado online (Gértrudix-Barrio; Rajas; Álvarez-García, 2017) y para la configuración curricular de cursos online como los massive open online courses (MOOC) o los small private online courses (SPOC). Dentro de los indicadores o criterios de calidad de estos cursos se incluye de forma destacada la creación de materiales audiovisuales (Aguaded; Medina-Salguero, 2015; Ramírez-Fernández; Salmerón-Silvera; López-Meneses, 2015).

El vídeo aplicado al proceso didáctico de enseñanza-aprendizaje presenta una serie de características y funciones intrínsecas que lo sitúan como uno de los componentes medulares de la denominada sociedad del conocimiento y como uno de los contenidos esenciales sobre el que se sustentan las metodologías didácticas en educación semipresencial y online (Gértrudix et al., 2017). Igualmente, se destaca como un instrumento activo de implicación o engagement del alumno en la materia abordada (Fischer et al., 2014; Guo; Kim; Rubin, 2014).

El vídeo forma parte del ecosistema digital de los medios de comunicación, de las redes sociales y de los universos virtuales de creación y consumo de contenidos a través de dispositivos tecnológicos que emplean docentes y alumnos. Es decir, conecta enseñanza, información, entretenimiento o marketing en un entorno abierto. Un mismo vídeo puede integrarse en el campus virtual de una asignatura, como un elemento exclusivo del itinerario curricular, o difundirse libremente en plataformas abiertas como YouTube (Welbourne; Grant, 2015), lo que genera valores añadidos de transferencia científica del conocimiento y de comunicación publicitaria para la institución universitaria de procedencia, por citar los dos principales (Burgess; Green, 2013; Rigutto, 2017; De-Lara; García-Avilés; Revuelta, 2017).
Tanto en el terreno del contenido científico (López-Meneses; Vázquez-Cano; Román-Graván, 2015) como en el ámbito del vídeo educativo en educación superior (White et al., 2013), los recursos audiovisuales se incorporan a metodologías de innovación didáctica como el aprendizaje basado en problemas (ABP/PBL) o la clase invertida (Bergmann; Sams, 2016), además de ser uno de los items esenciales en el itinerario formativo de cursos virtuales como los MOOCs o SPOCs (Hollands; Tirthali, 2014; Capdevilla-Pagès; Aranzadi-Elejabeitia, 2014; Hansch et al., 2015). En este sentido, el análisis de las nuevas formas de diseñar los contenidos y acceder a la información resulta un campo necesario de investigación (Sinha et al., 2014).

Dentro de los tipos y géneros que se encuentran en los vídeos educativos y científicos (Muñoz-Morcillo; Czurda; Robertson-von-Trotha, 2016) no ha sido suficientemente abordado, por novedoso, un formato que ha adquirido un papel relevante en el diseño de los cursos virtuales. Se trata del vídeo de presentación del curso o about video. ¿Qué convierte al vídeo de presentación de un MOOC en un objeto de estudio digno de atención? Precisamente su triple naturaleza comunicativa: poder ser a la vez un mensaje informativo, un contenido didáctico y un spot o tráiler publicitario.

El vídeo de presentación, 'acerca de' o about es un formato que los cursos $\mathrm{MOOC}$ mantienen fuera del propio itinerario de contenidos formativos del curso con la intención de dar a conocer distintos aspectos del mismo. Se difunde en dos espacios:

- la propia página de introducción o about del curso, donde acompaña a información textual, imágenes y enlaces para inscribirse en el MOOC;

- el ecosistema abierto de internet, incluyendo plataformas de vídeo streaming como YouTube, redes sociales como Twitter o Facebook y otros repositorios o páginas de difusión (bibliotecas virtuales y canales online de universidades, periódicos digitales, etc.).

El vídeo de presentación del MOOC se caracteriza, en consonancia con sus amplias posibilidades de diseminación, por la heterogeneidad de intenciones y resultados narrativos y estéticos que presenta. Se pueden encontrar tres funciones 
en su diseño narrativo:

- informar sobre determinados elementos del MOOC: tema, profesorado, metodología, funcionamiento, recursos bibliográficos o videográficos, etc.;

- adelantar contenidos didácticos o constituir una pieza formativa en sí misma: incluir una lección, un concepto, una clasificación, etc.;

- publicitar los valores sobresalientes del curso: motivos para inscribirse, ventajas diferenciales frente a otras propuestas, etc.

Del mismo modo, por definición los MOOCs van dirigidos a públicos objetivo muy variados y masivos, por lo que el alumnado o el perfil profesional

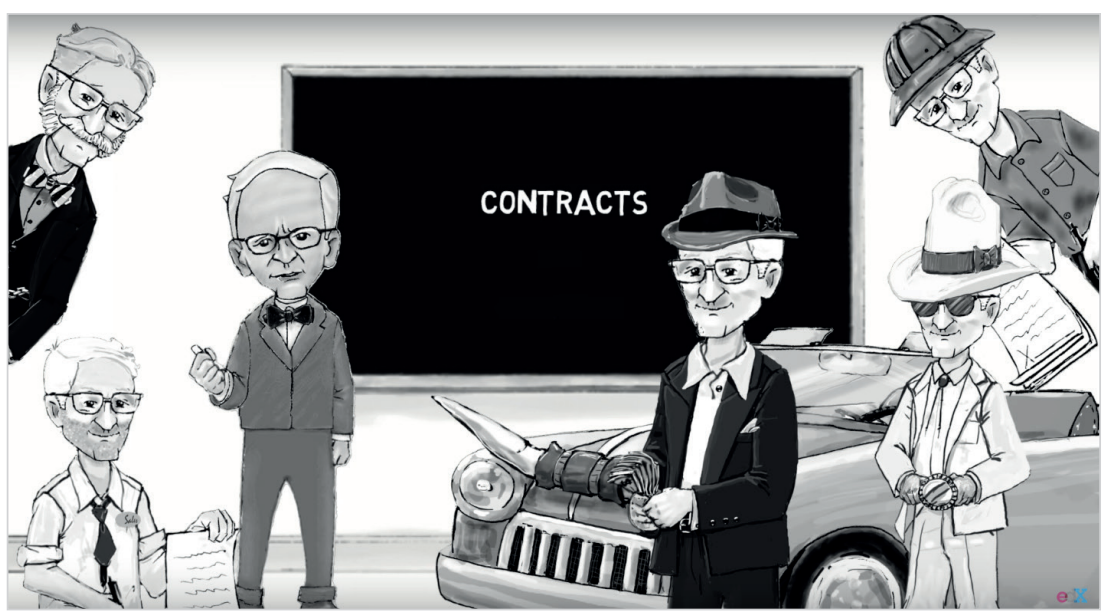

Imagen 1. Ilustraciones y animaciones exponen visualmente los contenidos didácticos. Contract law: From trust to promise to contract (HarvardX)

https://www.youtube.com/watch?v=7EyOXo8bRwU al que se orientan se convierte en un factor determinante en esa triple articulación del diseño del producto audiovisual en sentido informativo, didáctico o publicitario. Pocos formatos audiovisuales entroncan de forma tan manifiesta con el aprendizaje en la era digital: un ecosistema abierto de productos de formación, divulgación, información, entretenimiento y marketing. Se trata de un formato muy poco estudiado en profundidad, debido a que el vídeo didáctico en general ha sido abordado casi en exclusiva como objeto o recurso formativo, no como obra audiovisual que se integra en los medios de comunicación o en los canales de publicidad digital.

\section{Metodología}

Los objetivos de este análisis del vídeo de presentación o about como objeto informativo, didáctico y publicitario son:

- Conocer la estructura y contenido narrativo del formato.

- Estudiar los parámetros técnico-expresivos de realización audiovisual.

- Describir tendencias o tipos del formato.

- Valorar críticamente los resultados y proponer posibles mejoras en la calidad del formato.

La plataforma de MOOC seleccionada para el análisis es edX (https://www.edx.org). Se justifica la elección por los siguientes criterios:

- se trata de la segunda institución provider de MOOCs de educación superior con mayor número de usuarios registrados, 10 millones (Shah, 2016);

- es la segunda plataforma que incluye más universidades, organizaciones sin ánimo de lucro o empresas produciendo MOOCs de forma recurrente: a febrero de 2017, edX tiene más de 70 socios internacionales (Harvard y MIT la fundaron en 2012) que han lanzado a su vez 1.270 cursos.

La razón de no abordar el estudio de la plataforma más importante de MOOCs a nivel mundial, Coursera (https:// www.coursera.org), con 23 millones de usuarios registrados y 149 instituciones asociadas para un total de más de 2.000 cursos (DMR, 2017), es que no incluye vídeos de presentación o about fuera de la estructura del propio curso, sino incorporados al itinerario de contenidos de éste, lo que limita su impacto y le resta funciones comunicativas de marketing o publicidad.

La muestra seleccionada son 50 cursos MOOC de la plataforma edX. Un total de 50 páginas 'acerca de' o about, con posibles vídeos de presentación, elegidas aleatoriamente de entre los 244 cursos de $e d X$ en estado abierto o de inscripción a mayo de 2017 , lo que representa el 20,5\%.

\section{Formatos como vídeo o podcast son}

materiales recurrentes para los massive open online courses (MOOC), incluyéndose como indicadores o criterios de calidad de estos cursos

Los conjuntos de variables analizadas son:

- Aspectos generales: concurrencia o ausencia de vídeo en la página introductoria y duración de la pieza.

- Estructura formal: bloques y tipos de comienzo.

- Contenido narrativo: introducción, presentación de docentes, materia, metodología, funcionamiento interno, función publicitaria y otras marcas diferenciales.

- Realización audiovisual: videoclase, formato informativo o dramatización ficcional, rótulos o presentaciones visuales, animación o motion graphics, imagen dinámica, imagen estática, voz over y música, y marcas enunciativas de edición o montaje.

\section{Análisis y resultados}

\subsection{Aspectos generales}

De las 50 páginas about de MOOCs de edX que conforman la muestra, 33 contienen vídeo de presentación ${ }^{1}$ (66\%). Pertenecen a 26 instituciones: 23 universidades y 3 empresas.

Destaca la variedad del rango de duraciones, de las piezas más cortas (0:37, v17 y v46 del Anexo), a las de mayor extensión $(4: 22, v 16)$. La media es de 2:08 minutos por vídeo, pero no existe una homogeneización o estandarización en este sentido. Por lo tanto, en cuanto a la duración se apre- 
cia que existen piezas que siguen un formato tipo spot o anuncio y otras que optan por temporalidades más cercanas al reportaje en profundidad, la videoclase o al cortometraje.

\subsection{Estructura formal}

Respecto a la estructura, entendida como la organización de las partes distinguibles en cuanto a bloques diferentes de lenguajes, formatos o géneros audiovisuales (articular una videoclase con insertos tipo reportaje o motion graphics, por ejemplo), no respecto a sus líneas temáticas, los vídeos analizados contienen entre 1 ( $\sin$ bloques discernibles) y 4 componentes estructurales. Así, 11 vídeos sólo presentan una escena o secuencia continuada ( 8 de ellas, el $72 \%$, son videoclases: el profesor hablando a cámara en un fondo natural o entorno virtual tipo croma key); 12 contienen 2 partes; 7, 3 partes y sólo 3 vídeos contienen 4 partes, por lo que la fragmentación no es un rasgo distintivo de estas piezas, sino la continuidad que mantienen en su estructura formal, bastante uniforme y poco variable en su segmentación en posibles subunidades.

\section{El vídeo de presentación de un curso MOOC destaca por su triple naturaleza comunicativa: es a la vez un mensaje in- formativo, un contenido didáctico y un mensaje publicitario}

En cambio, destaca una relativa variedad de fórmulas de inicio de la pieza, un momento narrativo especialmente señalado en el vídeo didáctico como formato que busca impactar y llamar la atención del futuro alumno en el menor tiempo posible, poniendo en valor lo más destacado o reseñable del curso:

- 13 vídeos comienzan introduciendo el tema del MOOC: justifican su importancia y adelantan su contenido, sin dar lugar a otro tipo de expectativas generadas que no sean las de abordar el objeto de estudio del curso;

- 10 vídeos se inician con el docente que va a impartir el curso presentándose a cámara. Puede tratarse de una introducción muy breve (nombre, filiación y especialización), en cuyo caso se complementa con un índice o sumario de los contenidos a tratar en el vídeo, o de mayor extensión (como en v49) en el que el profesor hace un repaso de su currículo académico y trayectoria profesional como elementos de valor. Un vídeo (v4), no específicamente de presentación del docente al uso, añade una variante interesante: el profesor cuenta una historia de su vida, anécdotas y peripecias que acaban desembocando en la materia del curso;

- un tercer tipo es empezar lanzando una/s pregunta/s, es decir, planteando interrogantes que reclamen la atención y el interés del espectador. 9 de los vídeos optan por esta fórmula;
- una variante del comienzo de introducción temática es iniciar la pieza con un problema por resolver. Dos de los vídeos del primer grupo adoptan esta variante (v22 y v24).

\subsection{Contenido narrativo}

El análisis de los contenidos de cada vídeo arroja resultados relevantes en cuanto a la naturaleza informativa, educativa y publicitaria, que se señalaba como propia del formato. Se han contemplado las 6 siguientes variables:

- marco temático;

- presencia docente/s;

- contenidos del curso;

- metodología;

- funcionamiento interno (cronograma, evaluación, etc.);

- carácter publicitario del vídeo.

El $100 \%$ de los vídeos abordan el tema (de qué va a tratar el curso) como uno de los mensajes más importantes a comunicar. En todos se incluye una descripción, justificación o desarrollo del tema del MOOC. Puede parecer algo obvio tratándose de vídeos educativos, pero se detecta, en comparación con otras posibilidades narrativas (poner en valor la adquisición de competencias, enfocar la propuesta al alumno como usuario o público objetivo, diferenciarse de otros cursos, no en el contenido curricular sino en aspectos formales audiovisuales, etc.), una preeminencia del tema del curso como principal reclamo. En algunos vídeos, la mayor parte de la duración se consume en situar ese marco temático de la materia correspondiente.

En ese sentido, también los vídeos al completo explican, con mayor o menor profusión, los contenidos concretos que abarca el MOOC. Se pueden apreciar dos modelos, con gradaciones y combinaciones entre uno y otro:

- uno en el que se introduce un índice de lecciones generales o de epígrafes, más o menos exhaustivo;

- otro en el que no se mencionan unidades concretas sino líneas genéricas de contenido desarrolladas o derivadas del ámbito general que señalábamos más arriba.

En cuanto a la presencia de los docentes que van a impartir el curso, aunque mayoritariamente aparecen en los vídeos ( $85 \%$ del total), difiere la forma de introducirles en el rela- 
to y la importancia que les otorga, si bien en todos ellos el profesor es el personaje que va sirviendo de hilo conductor a los apartados.

Explicar o exponer la metodología empleada en el curso, tanto en lo referente a objetivos, actividades formativas o modelos docentes como al tipo de materiales utilizados (vídeos, documentos de texto y pruebas de evaluación tipo test, predominantemente), tiene una presencia sensiblemente menor: sólo en 14 vídeos (42,2\%), y en algunos ( 22 por ejemplo) sólo se hace referencia a los objetivos. Nuevamente se constata que el contenido determina o subordina a las posibilidades creativas y comunicativas de formatos como el MOOC: ningún vídeo hace referencia a metodologías innovadoras ni a la incorporación de novedades tecnológicas audiovisuales de la sociedad digital para impartir los contenidos de forma original o experimental.

\section{Los vídeos analizados sobresalen por la continuidad que mantienen en su es- tructura formal, bastante uniforme $y$ con pocas variaciones en su segmenta- ción en posibles subunidades}

La referencia al funcionamiento interno del curso apenas tiene presencia en 12 de las propuestas (36,3\%). Se alude, mayoritariamente, a las semanas en las que se imparte y a las horas aproximadas de dedicación, y, por otro lado, a criterios generales de evaluación, sin detallar información demasiado compleja o estricta que pueda llegar a desincentivar al alumno.

Finalmente, un análisis fundamental consiste en dilucidar si los vídeos de presentación muestran rasgos publicitarios, como herramienta de marketing que busca captar alumnos para su inscripción en el curso, y cómo llevan esta función a cabo. 17 de las piezas (51,5\%) muestran explícitamente su carácter persuasivo, haciendo evidente su naturaleza de spot, tráiler o publirreportaje, sobre todo a través del lenguaje verbal empleado por los profesores o las voces over de narradores que invitan a apuntarse al curso, lo destacan entre otros o hablan de los beneficios académicos o profesionales que los alumnos van a obtener con él. Todos ellos, en cabeceras y componentes de diseño gráfico, hacen referencia a la identidad visual de las instituciones generando valores de imagen corporativa (HarvardX, MITx, ColumbiaX, PekingX, etc.).

Sin embargo, aunque no todos los vídeos manifiesten de modo evidente que están emparentados con formatos de marketing audiovisual, indirectamente el propio vídeo de presentación o about contiene un mensaje publicitario por definición: se vende la participación en un curso. De ahí que predomine, en gran parte de ellos, la justificación de la importancia del tema del MOOC, la exposición de ventajas diferenciales respecto a la oferta de cursos o la presentación del profesorado, aunque no se transmita esta información persuasiva como si se tratara de publicidad sino de contenido informativo.

Esto implica dos elementos de interés:

- los objetos de aprendizaje en formato de vídeo didáctico (una videoclase, una entrevista, el registro de un experimento, por ejemplo) al pertenecer a una estructura o programación más amplia (el $\mathrm{MOOC}$ ) y a una institución universitaria o empresarial que funciona como productora de los recursos audiovisuales, puede enfocarse, por acción o por omisión, como un contenido publicitario del curso y de otras propuestas formativas que emprendan dichas organizaciones;

- todos los vídeos analizados pertenecen a las marcas específicas que las instituciones han lanzado para crear los cursos (HarvardX, MITX, ColumbiaX, PekingX, etc.) y que quedan representadas en cabeceras, títulos, logotipos $y$, en definitiva, en la imagen corporativa que proyectan los vídeos al seguir una identidad visual similar a nivel de grafismo, no de realización audiovisual, salvo algunas excepciones como la UPValenciaX, que sigue un estilo "polimedia" similar en todos los casos analizados. Este formato de vídeo (media.upv.es) se caracteriza por automatizar procesos de grabación y edición en directo y por consistir en un diseño visual integral compuesto por el docente en la derecha del encuadre y una presentación tipo Power Point ocupando la mayor parte de la pantalla.

Del mismo modo, resultan de interés las marcas diferenciales de los vídeos. Es decir, no lo que tienen en común, sino los elementos distintivos de contenido que incorporan. Las más destacadas son:

- hablar de los requisitos para acceder al curso (v2 y v28);

- citar bibliografía (v12 y v39);

- introducir la opinión de los alumnos (v13);

- formular problemas a resolver durante el curso (v18);

- contar quiénes son los profesores en la locución, dándole un carácter más publicitario (v23) o incluir la participación de muchos (v40 y v41);

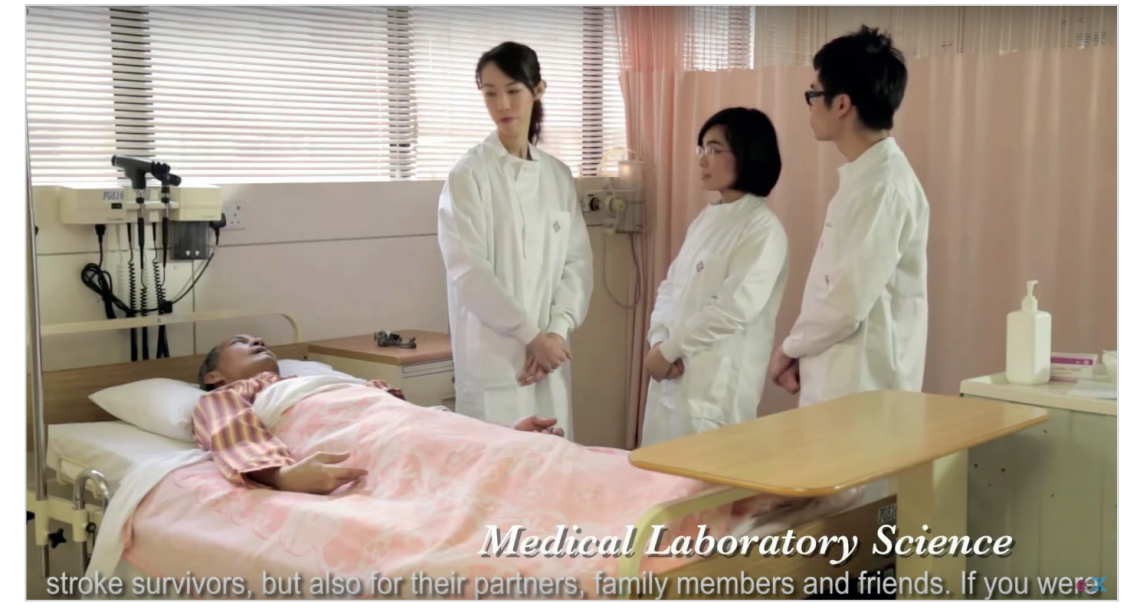

Imagen 3. Los formatos de ficción permiten la convergencia de la formación y el entretenimiento. Human anatomy (HKPolyUx)

https://www.youtube.com/watch?v=_F9BtdWqMg 


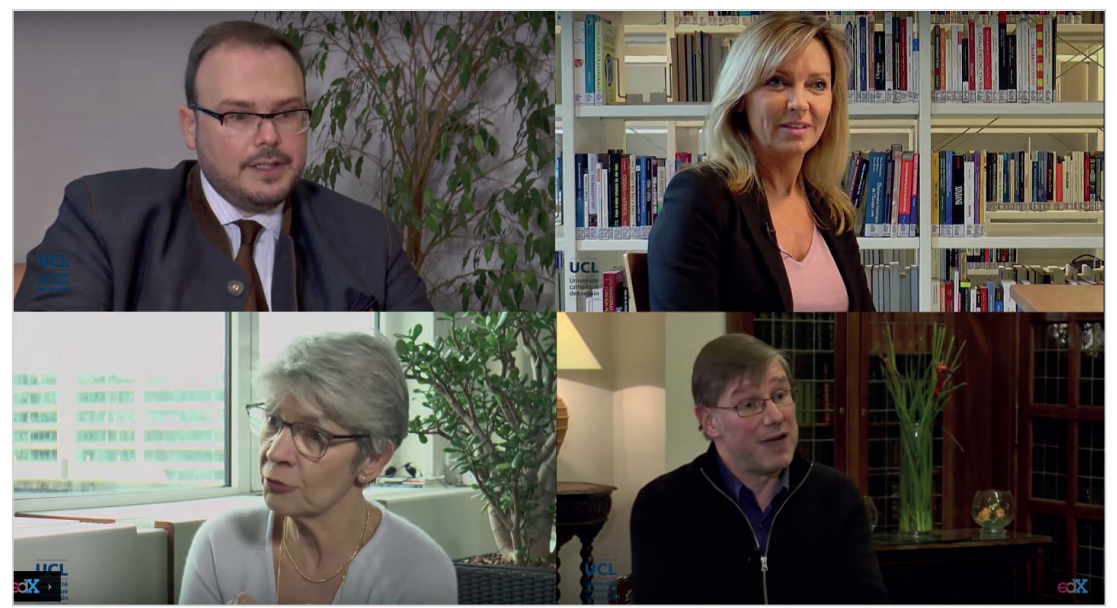

Imagen 4. Los recursos audiovisuales amplían las posibilidades didácticas de los MOOCs. Découvrir la science politique (LouvainX)

https://www.youtube.com/watch?v=uMdICJTyuNA

- comentar éxitos anteriores (v32);

- conectar el curso presente con otros cursos (v35);

- finalizar con más preguntas (v38) o añadiendo un mensaje motivador final (v30).

\subsection{Realización audiovisual}

Se han analizado las sustancias expresivas visuales y sonoras utilizadas de acuerdo con los siguientes parámetros:

- puesta en escena: formato tipo videoclase en el que el profesor habla a cámara;

- relato informativo o dramatizado: entrevistas, reportajes o elementos ficcionales;

- diseño gráfico audiovisual: rótulos, títulos o presentaciones visuales tipo Power Point;

- animación: gráficos en movimiento o animación 2D/3D;

- imagen dinámica: planos de vídeo;

- imagen estática: fotografías, ilustraciones, tablas, etc.;

- música: banda sonora;

- voz over: narración de locutor.

27 de los 33 vídeos $(81,8 \%)$ presentan una puesta en escena basada en un docente que cuenta al espectador (cámara) el contenido narrativo del vídeo. Como se ha señalado, es una escenificación que procede de identificar la clase presencial con una supuesta lección audiovisual.

Sólo 5 de los $33(15,15 \%)$ introducen componentes audiovisuales propios de relatos informativos (4 incluyen bloques semejantes a entrevistas y reportajes televisivos: v3, v24, v31 y v33) y un único dramatizado (v45 y sólo en la parte ficcional en la que muestran escenas en un hospital).

En cuanto a la utilización de elementos de diseño gráfico audiovisual, destaca la introducción de títulos y cabeceras en el $100 \%$ de la muestra y de rótulos o lower thirds y presentaciones visuales en 22 de los 33 vídeos (66,6\%). En este apartado hay mucha disparidad formal, muchos estilos $y$ niveles de calidad, pero, en general, resultan bastante eficaces, visualmente atractivos y cumplen su función informativa, expresiva y estética.
Menor es la presencia de técnicas de animación, siendo un género con destacadas cualidades narrativas en el terreno del vídeo educativo. La utilización de motion graphics y animación 2D/3D se encuentra en 10 piezas $(30,3 \%)$ y la factura visual bascula desde el uso de plantillas y presets más o menos modificados (v24 y v41) a la elaboración de animación personalizada (v49).

Respecto a la utilización de imagen dinámica, es decir, la presencia de vídeo, 21 ejemplos $(63,6 \%)$ contienen planos de este tipo, bien como imágenes de archivo, bien grabadas para la pieza, destacando la función descriptiva o informativa de las imágenes (planos inserto o recurso) sobre la intencionalidad narrativa o dramática.

En ese mismo sentido, la imagen estática (ilustraciones, fotografías, dibujos, tablas, etc.) se encuentra presente en 16 vídeos $(48,4 \%)$, una cantidad muy alta en comparación con formatos de vídeo convencionales. Nuevamente, esto es debido a que se plantea el about como un vídeo deudor de las clases presenciales y la utilización de presentaciones gráficas estáticas más que de una forma de comunicar propia de los medios audiovisuales.

Finalmente, en el aspecto sonoro, destaca la utilización de música en 23 vídeos $(69,6 \%$ ) y la concurrencia de voz over (la narración locutada) en 19 de las piezas analizadas (57,5\%). La articulación de los elementos de la banda sonora resulta de especial relevancia, ya que la información transmitida en estos vídeos depende más del audio que de la imagen, por esa cercanía genérica con la clase presencial que apuntábamos anteriormente. Ni uno sólo de los vídeos prescinde de la palabra hablada, bien con el personaje en pantalla, bien en off u over, para comunicar su mensaje informativo, didáctico o publicitario.

\section{A pesar de estar bien enfocados en} cuanto a los objetivos de dar a conocer, enseñar y publicitar el MOOC, los vídeos no alcanzan un nivel adecuado de realización audiovisual

Dando un paso más en el análisis, de las 33 piezas estudiadas, ninguna presenta los 8 componentes analizados; sólo 3 ( $v 22, v 23$ y v24) contienen 7 de ellos (a las dos primeras les falta el relato informativo o dramatizado y a la tercera la imagen fija); 7 vídeos contienen 6 (v32, v38, v39, v40, v44, v45 y v49); y, en el otro extremo, 3 vídeos emplean sólo 2 de los componentes audiovisuales (v2, v10 y v17), el primero y el tercero limitándose a la puesta en escena del profesor en pantalla y la inclusión de grafismo, y el segundo a la puesta en escena y la presencia en montaje paralelo de imágenes 
fijas, sin elementos de diseño gráfico añadidos. No hay ninguna pieza que sólo haya empleado uno de los componentes analizados.

Al agrupar las variables, no se encuentran correspondencias evidentes entre las sustancias expresivas puestas en escena. Esto permite constatar que no se trata de vídeos pensados desde un diseño integral de la realización audiovisual, en la que determinados componentes guardan una relación más estrecha entre contenido y forma o entre historia y discurso y, además, existen vinculaciones reconocibles o fórmulas tipificadas en la gramática audiovisual entre los lenguajes visuales y sonoros. En estos vídeos, parece que los materiales técnico-expresivos se introducen más en sentido aleatorio que siguiendo un plan de diseño de realización audiovisual específico.

\section{Conclusiones}

El análisis del diseño informativo, didáctico y publicitario del vídeo de presentación o about de la plataforma edX que hemos llevado a cabo permite detectar las siguientes tendencias.

En primer lugar, se trata de producciones que, a pesar de estar bien enfocadas en cuanto a los objetivos de dar a conocer, enseñar ya en cierta medida y publicitar el MOOC, no alcanzan un nivel notable en cuanto a la realización audiovisual. Si analizamos el contenido, el guion narrativo de las piezas, no se encuentran estrategias ni técnicas narrativas de las habitualmente utilizadas en televisión o cine. Este hecho redunda en que, salvo en el potencial de las temáticas o de los docentes, podría resultar difícil conectar con el espectador a nivel de atención o de emoción, al no recurrir a mecanismos narrativos en el relato que le involucren.

\section{En los vídeos analizados subyace un diseño que tiene su origen en el vídeo didáctico que procede del registro de la clase presencial}

La utilización de recursos visuales y sonoros no responde a un diseño de producción audiovisual (interpretación, fotografía, sonido, montaje, efectos visuales) que asemeje el formato de vídeo de presentación o about a un spot, un videoclip, un reportaje o un cortometraje de ficción donde el ejercicio de la realización consiste, fundamentalmente, en integrar esos lenguajes en un sistema interconectado, coherente y significativo.

Esto es debido a que en los productos analizados subyace un diseño que tiene su origen en el vídeo didáctico en sentido convencional: el que procede del registro de la clase presencial. Los ejemplos analizados parecen, salvo alguna excepción, lecciones magistrales grabadas en escenografías naturales o virtuales, ilustradas con imágenes fijas y en movimiento y acompañadas de músicas y locuciones. Por eso no hay un diseño de realización audiovisual en el que puedan percibirse intencionalidades evidentes de cara a influir emocionalmente en el espectador y se constata que más que adaptar a imágenes y sonidos contenidos didácticos de forma optimizada, sencillamente se han grabado contenidos y se han trasladado con pocas modificaciones a la pantalla.

De los análisis realizados, por el contrario, se pueden extraer posibles mejoras o implementaciones en el diseño informativo, didáctico y publicitario de estos vídeos. Estos avances narrativos y estéticos buscan potenciar la triple naturaleza del formato. Se señalan:

- En sentido de estrategia de comunicación educativa y de marketing, el vídeo de introducción se plantea como un contenido autónomo que aporte valor en sí mismo y que pueda difundirse desligado de la página de introducción al curso. Es decir, que tenga utilidad para el espectador-alumno y de esa manera generar impacto y difusión para cumplir determinados objetivos didácticos y publicitarios.

- Construir el relato informativa y emocionalmente: no sólo introducir el tema general, el docente, el itinerario, la metodología o el funcionamiento del curso, sino incorporar técnicas narrativas (progresión, giros, reveses, flashbacks, etc.) para que el contenido tenga interés y produzca conexión o engagement con el espectador-alumno.

- Cuidar el lenguaje audiovisual trasladando los códigos de la televisión, el cine o la radio a las propuestas de vídeo didáctico.

- Crear imágenes de marca: incluir identidades visuales de las universidades no sólo en las cabeceras o los títulos sino en la forma de contar una historia, dejando libertad creativa para cada MOOC en concreto, pero haciendo que cada curso remita al conjunto de las propuestas formativas de la institución tanto en el contenido como en la forma.

- Elaborar propuestas de formato más acordes con la difusión o transmisión del conocimiento en entornos virtuales. Crear vídeos más cortos o versiones menos extensas 
de las piezas originales para redes sociales (Twitter, Instagram, Facebook, etc.) y plataformas de internet (YouTube y Vimeo, principalmente), así como dejar que los alumnos realicen sus propios materiales para dar a conocer el curso.

En relación con las investigaciones precedentes, se constata que, si bien se están produciendo análisis muy relevantes en el campo del contenido temático, todavía resultan escasas las investigaciones sobre realización audiovisual aplicada a este tipo de materiales videográficos desde el punto de vista técnico y expresivo que conduzcan a mejorar y profesionalizar la creación de estos contenidos formativos.

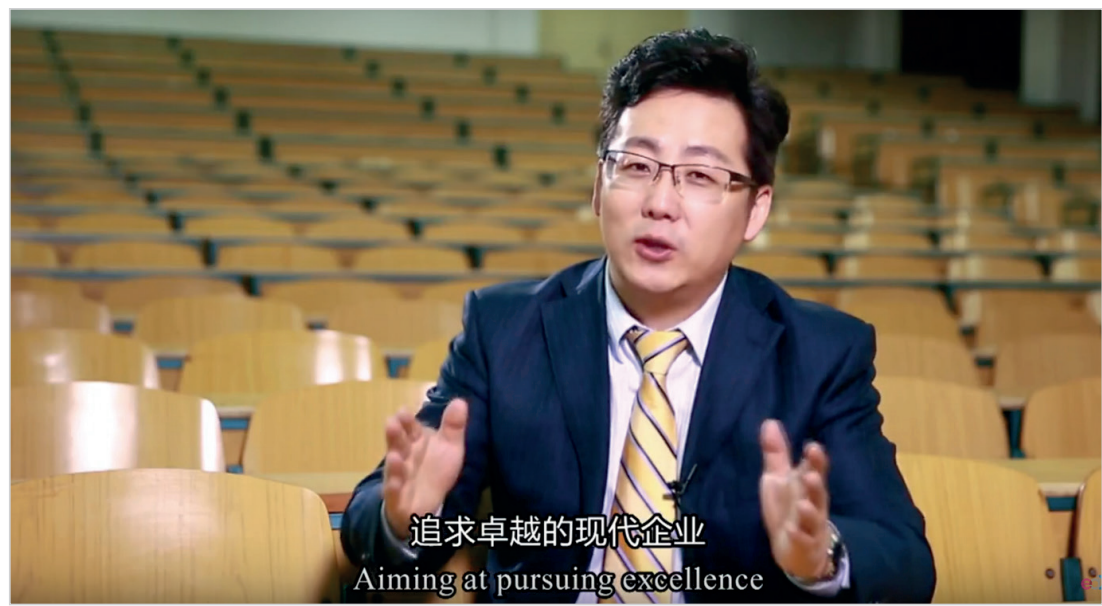

Imagen 6. Entrevistas, debates o reportajes incorporan técnicas narrativas televisivas al proceso de aprendizaje. Management communication (PekingX)

https://www.youtube.com/watch?v=afPmZ8A7SBo
El vídeo de presentación o about es un formato que abre el camino a generar recursos educativos que puedan ser comunicados en entornos virtuales amplios y cumplir objetivos didácticos, informativos o publicitarios que redunden tanto en el éxito del MOOC como en la estrategia global de la institución, además de ayudar a generar y actualizar la comunidad educativa abierta y permanente en el que se ha convertido el ecosistema de la Red.

\section{Se debería construir el relato informati- va y emocionalmente, incorporando téc- nicas narrativas para que el contenido tenga interés y produzca engagement con el espectador-alumno}

\section{Nota}

1. Al final del documento se encuentra el listado de los vídeos analizados. Debido a que la mayoría presentan denominaciones muy largas, se citan por su orden en esa lista (vx) para facilitar la lectura.

\section{Referencias}

Aguaded, Ignacio; Medina-Salguero, Rosario (2015). “Criterios de calidad para la valoración y gestión de MOOC". RIED. Revista iberoamericana de educación a distancia, v. 18, n. 2, pp. 119-143.

https://doi.org/10.5944/ried.18.2.13579

Bergmann, Jonathan; Sams, Aaron (2016). Dale la vuelta a tu clase. Lleva tu clase a cada estudiante, en cualquier momento y en cualquier lugar. Madrid: SM. ISBN: 9788467561180

Burgess, Jean; Green, Joshua (2013). YouTube: Online video and participatory culture. Nueva York: John Wiley \& Sons. ISBN: 9780745644790

Capdevilla-Pagès, Ramón; Aranzadi-Elejabeitia, Pedro (2014). "Los cursos online masivos y abiertos: ¿oportunidad o amenaza para las universidades iberoamericanas?". RIED. Revista iberoamericana de educación a distancia, v. 17, n. 1, pp. 69-82. https://doi.org/10.5944/ried.17.1.11574
$D M R$ (2017). "19 interesting Coursera stats and facts". DMR Business statistics fun gadgets.

http://expandedramblings.com/index.php/businessdirectory/19718/coursera

De-Lara, Alicia; García-Avilés, José-Alberto; Revuelta, Gema (2017). "Online video on climate change: a comparison between television and web formats". JCOM. Journal of science communication, v. 16, n. 1, A04.

https://jcom.sissa.it/archive/16/01/JCOM_1601_2017_A04

Fischer, Helge; Dreisiebner, Stefan; Franken, Oliver; Ebner, Martin; Kopp, Michael; Koehler, Thomas (2014). "Revenue vs. costs of MOOC platforms. Discussion of business models for XMOOC providers, based on empirical findings and experiences during implementing the project iMooX". En: Iceri2014, $7^{\text {th }}$ Intl conf of education, research and innovation, pp. 2991-3000. Valencia: lated Academy, SL. ISBN: 978 8461724840

https://goo.gl/KBM5zr

Gértrudix-Barrio, Manuel; Rajas-Fernández, Mario; Álvarez-García, Sergio (2017). “Metodología de producción para el desarrollo de contenidos audiovisuales y multimedia para MOOC". RIED. Revista iberoamericana de educación a distancia, v. 20, n. 1, pp. 183-203.

https://doi.org/10.5944/ried.20.1.16691

Gértrudix-Barrio, Manuel; Rajas-Fernández, Mario; Barrera-Muro, Daniel; Bastida, María; Soto, Cynthia (2017). "Realización de vídeo educativo: análisis de la producción audiovisual de los MOOC de URJCx". En: Sierra-Sánchez, Javier (coord.). Nuevas tecnologías audiovisuales para nuevas narrativas interactivas digitales en la era multidispositivo. Madrid: McGraw Hill Education, pp. 289-302. ISBN: 97884 48613594 https://goo.gl/h9YTdM

iSpring (2016). 6 tips to create engaging training videos. https://www.ispringsolutions.com/blog/6-tips-to-createengaging-training-videos

Kaltura (2016). "The state of video in education". https://goo.gl/iKNuO5 
Guo, Philip; Kim, Juho; Rubin, Rob (2014). “How video production affects student engagement: An empirical study of MOOC videos". Proceedings of the first ACM conf on learning@scale conf, pp.41-50.

http://dx.doi.org/10.1145/2556325.2566239

Hansch, Anna; Hillers, Lisa; McConachie, Katherine; Newman, Christopher; Schildhauer, Thomas; Schmidt, Philipp (2015). "Video and online learning: Critical reflections and findings from the field". HIIG discussion paper series, $\mathrm{n}$. 2015-02, pp. 1-34.

https://doi.org/10.2139/ssrn.2577882

Hollands, Fiona M.; Tirthali, Devayani (2014). "Why do institutions offer MOOCs?". Online learning: Official journal of the online learning consortium, v. 18, n. 3.

https://doi.org/10.24059/olj.v18i3.464

López-Meneses, Eloy; Vázquez-Cano, Esteban; Román-Graván, Pedro (2015). "Análisis e implicaciones del impacto del movimiento MOOC en la comunidad científica: JCR y Scopus (2010-13)". Comunicar, v. 22, n. 44.

https://doi.org/10.3916/C44-2015-08

Muñoz-Morcillo, Jesús; Czurda, Klemens; Robertson-von-Trotha, Caroline Y. (2016). "Typologies of the popular science web video". JCOM. Journal of science communication, v. 15, n. 4, A02.

https://jcom.sissa.it/sites/default/files/documents/ JCOM_1504_2016_A02.pdf

Ramírez-Fernández, Miguel-Baldomero; Salmerón-Silvera, José-Luis; López-Meneses, Eloy (2015). “Comparativa entre instrumentos de evaluación de calidad de cursos MOOC". RUSC, v. 12, n. 1, pp. 131-151.

https://doi.org/10.7238/rusc.v12i1.2258
Rigutto, Cristina (2017). "The landscape of online visual communication of science". JCOM. Journal of science communication, v. 16, n. 2, C06.

https://goo.gl/3xEnPs

Shah, Dhawal (2016). "By the numbers: MOOCs in 2016. How has the MOOC space grown this year? Get the facts, figures, and pie charts". Class central, 25 December.

https://www.class-central.com/report/mooc-stats-2016

Sinha, Tanmay; Jermann Patrick; Li, Nan; Dillenbourg, Pierre (2014). "Your click decides your fate: Inferring information processing and attrition behavior from MOOC video clickstream interactions". En: Procs of the 2014 Conf on empirical methods in natural language processing (Emnlp), October 25-29, Doha, Qatar, pp. 3-14.

http://www.ac/web.org/anthology/W14-4102

Welbourne, Dustin J.; Grant, Will J. (2015). "Science communication on YouTube: Factors that affect channel and video popularity". Public understanding of science, v. 25, n. 6, pp. 706-718.

https://goo.gl/AU1DAj

https://doi.org/10.1177/0963662515572068

White, Timothy J.; Ran, Low H.; Hou, Roy N. J.; Othman, Bin; Ridwan, Mohamad (2013). "MOOCs: A diminution, evolution or revolution in university teaching?". IEEE 63 ${ }^{\text {rd }}$ Annual conf Intl Council for Educational Media (ICEM). ISBN: 9781479932160

https://goo.gl/usDDkS

https://doi.org/10.1109/CICEM.2013.6820238

Woolfitt, Zac (2015). The effective use of video in higher education. Amsterdam: Inholland University of Applied Sciences. https://goo.gl/bhdju5

Anexo

\begin{tabular}{|c|c|c|c|c|c|}
\hline & Título del vídeo & Institución & Vídeo & URL & Duración \\
\hline v1 & Windows server 2016 virtualization & Microsoft & Sí & https://www.youtube.com/watch?v=8GJQOnNwRyw & $0: 39$ \\
\hline $\mathrm{v} 2$ & Bases matemáticas: integrales & UPValenciaX & Sí & https://www.youtube.com/watch?v=gugR5p 1 wR8k & $1: 50$ \\
\hline v3 & Reacciones químicas y cálculos estequiométricos & UPValenciaX & No & & \\
\hline v4 & $\begin{array}{l}\text { Finance for everyone: Smart tools for deci- } \\
\text { sion-making }\end{array}$ & MichiganX & Sí & https://www.youtube.com/watch?v=nNaP4mHIMc8 & 3:09 \\
\hline v5 & $\begin{array}{l}\text { Anatomy: Musculoskeletal and integumentary } \\
\text { systems }\end{array}$ & MichiganX & No & & \\
\hline v6 & Shylock's bond & HarvardX & Sí & https://www.youtube.com/watch?v=l-ppWDIRJho & $0: 45$ \\
\hline v7 & Deep learning explained & Microsoft & No & & \\
\hline v8 & $\begin{array}{l}\text { Developing business applications with power } \\
\text { Apps and Microsoft flow }\end{array}$ & Microsoft & No & & \\
\hline v9 & Predictionx: diviner's guid & HarvardX & No & & \\
\hline v10 & Tecnologías para la educación & UPValenciaX & Sí & https://www.youtube.com/watch?v=vPJURpuxVEo & $1: 18$ \\
\hline v11 & How to code: Complex data & $U B C x$ & No & & \\
\hline v12 & Autonomous mobile robots: Amrx & $E T H x$ & Sí & https://www.youtube.com/watch?v=RyMzGMpLpjk & $3: 00$ \\
\hline v13 & Becoming an entrepreneur & MITx & Sí & https://www.youtube.com/watch?v=C4KNrKSx-zE & $0: 48$ \\
\hline
\end{tabular}




\begin{tabular}{|c|c|c|c|c|c|}
\hline v14 & Evidence-based stakeholder marketing & ANUX & No & & \\
\hline v15 & UX design: From wireframe to prototype & MichiganX & No & & \\
\hline v16 & Introducción a la gestión de proyectos & UPValenciaX & Sí & https://www.youtube.com/watch?v=rg1gHs5Mw7A & $4: 22$ \\
\hline v17 & $\begin{array}{l}\text { Windows Server 2016: Azure for on-premises } \\
\text { administrators }\end{array}$ & Microsoft & Sí & https://www.youtube.com/watch?v=08TK6Q06iE8 & $0: 37$ \\
\hline v18 & Aplicaciones de la Teoría de grafos a la vida real II & UPValenciaX & Sí & https://www.youtube.com/watch?v=RoJBjqRbRvY & $3: 00$ \\
\hline v19 & Excel & UPValenciaX & Sí & https://www.youtube.com/watch?v=1SwaTMeKyBY & $1: 02$ \\
\hline v20 & Introduction to Python: Fundamentals & Microsoft & No & & \\
\hline v21 & Marketing fundamentals: who is your customer? & BabsonX & Sí & https://www.youtube.com/watch?v=K11iA7RYAWI & 2:06 \\
\hline $\mathrm{v} 22$ & Sustainable food security: crop production & Wageningen $X$ & Sí & https://www.youtube.com/watch?v=dsXo1uNOxN8 & 2:37 \\
\hline v23 & $\begin{array}{l}\text { Sustainable food security: The value of systems } \\
\text { thinking }\end{array}$ & Wageningen $X$ & Sí & https://www.youtube.com/watch?v=RsueubsgKUA & $1: 30$ \\
\hline v24 & Management communication & PekingX & Sí & https://www.youtube.com/watch?v=afPmZ8A7SBo & 2:15 \\
\hline v25 & Introduction to cybersecurity & UWashingtonX & No & & \\
\hline v26 & Introduction to Apache hadoop & LinuxFoundationX & No & & \\
\hline v27 & Fundamentals of red hat enterprise Linux & Red Hat & Sí & https://www.youtube.com/watch?v=MQRiT9BQDVQ & $1: 20$ \\
\hline v28 & Dispositivos móviles para la gestión del territorio & UPValenciaX & Sí & https://www.youtube.com/watch?v=XzLspS9cAq0 & 3:51 \\
\hline v29 & $\begin{array}{l}\text { Public library management: Managing a diverse } \\
\text { and inclusive workplace }\end{array}$ & MichiganX & No & & \\
\hline v30 & Principles of biochemistry & HarvardX & Sí & https://www.youtube.com/watch?v=AoZAiWMwe50 & $1: 10$ \\
\hline v31 & $\begin{array}{l}\text { Organizing evangelism and achieving user } \\
\text { adoption }\end{array}$ & Microsoft & No & & \\
\hline v32 & Reinvent yourself: Unleash your creativity & UTHealthSPHx & Sí & https://www.youtube.com/watch?v=U52UoJcmfxY & $1: 20$ \\
\hline v33 & Introduction to managerial economics & IIMBX & Sí & https://www.youtube.com/watch?v=7UF4yZ8VejU & $2: 22$ \\
\hline v34 & Innovation and IT management & IIMBX & Sí & https://www.youtube.com/watch?v=j-e_Vj3Frok & $1: 49$ \\
\hline v35 & $\begin{array}{l}\text { Saving schools: Reforming the U.S. education } \\
\text { system }\end{array}$ & HarvardX & Sí & https://www.youtube.com/watch?v=LYP6UkLtmxY & $3: 11$ \\
\hline v36 & Advanced transport phenomena & DelftX & Sí & https://www.youtube.com/watch?v=pKbl_01ds24 & $2: 21$ \\
\hline v37 & Introduction to typescript 2 & Microsoft & No & & \\
\hline v38 & $\begin{array}{l}\text { Foundations of data analysis. Part 1: Statistics } \\
\text { using } R\end{array}$ & UTAustinX & Sí & https://www.youtube.com/watch?v=LXfoZGmg-rE & $2: 31$ \\
\hline v39 & $\begin{array}{l}\text { Women have always worked: the U.S. Experi- } \\
\text { ence 1920-2016 }\end{array}$ & ColumbiaX & Sí & https://www.youtube.com/watch?v=dLnvAXUpMRo & 3:50 \\
\hline $\mathrm{v} 40$ & Découvrir la science politique & LouvainX & Sí & https://www.youtube.com/watch?v=uMdICJTyuNA & 2:37 \\
\hline v41 & Communicating corporate social responsibility & LouvainX & Sí & https://www.youtube.com/watch?v=PvVMESAMX1M & $3: 17$ \\
\hline $\mathrm{v} 42$ & Enhancing your career competencies & PekingX & No & & \\
\hline v43 & $\begin{array}{l}\text { Hábitos de consumo sostenible: motor de } \\
\text { innovación }\end{array}$ & JaverianaX & Sí & https://www.youtube.com/watch?v=3dfkGcJylrg & $2: 11$ \\
\hline v44 & Research methods: an engineering approach & WitsX & Sí & https://www.youtube.com/watch?v=umM1DN261iw & $2: 02$ \\
\hline v45 & Human anatomy & HKPolyUx & Sí & https://www.youtube.com/watch?v=_-F9BtdWqMg & $2: 45$ \\
\hline v46 & On Ramp to $A P^{*}$ Biology & WestonX & Sí & https://www.youtube.com/watch?v=SMEJuHJf9vo & $0: 37$ \\
\hline v47 & Cybersecurity: the ciso's view & UWashingtonX & No & & \\
\hline v48 & $\begin{array}{l}\text { Understanding the Australian health care } \\
\text { system }\end{array}$ & Uqx & No & & \\
\hline v49 & Contract law: From trust to promise to contract & HarvardX & Sí & https://www.youtube.com/watch?v=7EyOXo8bRwU & $2: 38$ \\
\hline v50 & Journalism for social change & UCBerkeleyX & Sí & https://www.youtube.com/watch?v=_pCs9e-iAuY & $1: 45$ \\
\hline
\end{tabular}

